\title{
Application of Anisotropic Vector Preisach Model for Bulk Materials
}

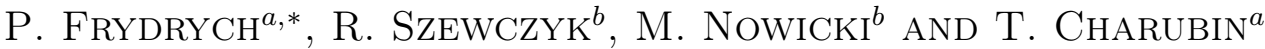 \\ ${ }^{a}$ Institute of Metrology and Biomedical Engineering, Warsaw University of Technology, Warsaw, Poland \\ ${ }^{b}$ Research Institute for Automation and Measurements, Warsaw, Poland
}

\begin{abstract}
In this paper possibility of application two-dimensional vector Preisach model for bulk materials was investigated. Physical magnetization mechanisms in bulk cores and thin ribbons were analyzed. Model is based on collection of the Preisach planes which describe material state in different angles on rotation plane. Presented model exhibits good conformity with experimental data for bulk as well for ribbon shaped cores. Model includes anisotropy and describes not only mean magnetization vector, but also distribution of magnetic moments for different angles.
\end{abstract}

DOI: 10.12693/APhysPolA.131.618

PACS/topics: 75.60.-d, 75.60.Ch, 75.60.Nt, 75.30.Gw, 85.70.--w

\section{Introduction}

Analysis of distribution of magnetization in material can be used for optimization of magnetic cores of sensors and power conversion devices [1]. Nonetheless magnetization distribution can be also used to estimate internal stress, tensile and crack evaluation, which plays crucial role in nondestructive testing [2]. Especially in nondestructive testing methods based on magnetic properties measurement, direction of magnetic moments in material and local stress inducted anisotropy is not obvious. Presented model enables investigation of material magnetic parameters for different angles of magnetization. It can be used as reference data for other analysis techniques such as [3].

\section{Magnetization process in bulk materials}

In bulk materials (ferrites are examples thereof) magnetization process is governed by the movement of $180^{\circ}$ domain walls (the Bloch walls). Less important are the so called Néel walls, which on the other hand are crucial for thin films and ribbons that can be approximated by two-dimensional domain structure model such as the Van den Berg construction [4]. Distribution of magnetic moments in thin cores was simulated in [5] and [6]. For both domain wall types external magnetic field vector and domain magnetization vector are on the same rotation plane. For that reason for isotropic materials such as ferrites and materials with uniaxial anisotropy two-dimensional magnetic moments model can be good approximation of three-dimensional material. Presented model can be related with theory of irreducible representations of the group of rotations proposed by Mayergoyz [7]. The difference is that author of this paper uses physical processes such as domain wall movement and domain rotation instead of mathematical theory to solve the identification problem.

*corresponding author; e-mail: p.frydrych@mchtr.pw.edu.pl

\section{Two-dimensional magnetization model}

Single Preisach plane describes distribution of switching operators for increase of and decrease of magnetic field for only one direction. It assumes that magnetic spins can only be oriented accordant or opposite, but still in the same direction. Distribution can be calculated from set of hysteresis loops for different field amplitudes. Distribution can be also fitted using two-dimensional Gaussian or other probability density function. For many applications apart from some amorphous alloys [8] Gaussian distribution seems to be insufficient. Best results were achieved for Lorentzian curve [9]. For two-dimensional model Gaussian probability density function gave good conformity with measured data [5]. There were three reasons why good conformity for Gaussian distribution was achieved. First reason was that there were two magnetization mechanisms introduced. Second, single Preisach plane for real magnetic cores apart from magnetic wires should represent not only one direction of magnetization but also projection of other directions. Third reason was that the anisotropy as factor influencing on distribution shape for different angles was introduced. For two-dimensional Preisach model mean magnetization is a sum of magnetization vectors for each angle (1). Integral on the Preisach plane can have positive or negative values. Thus vector $-\boldsymbol{m}$ for angle $\theta$ is the same as vector $\boldsymbol{m}$ for angle $\theta+\pi(2)$ :

$$
\boldsymbol{M}=\int_{0}^{2 \pi} \boldsymbol{m}(\theta) \cos \theta \mathrm{d} \theta .
$$

For every angular homogeneous plane two opposite Preisach planes exist. They represent domains oriented in the same angle but with opposite values.

\subsection{Extension to three dimensions}

Two-dimensional model can be easily extended to three dimensions. During magnetization process spins, only change their $\theta$ angle. For two dimensions there is only one $\lambda$ value. When material is isotropic for all $\lambda$ values

$$
\boldsymbol{M}=\int_{0}^{2 \pi} \mathrm{d} \lambda \int_{0}^{2 \pi} \boldsymbol{m}(\theta, \lambda) \cos \theta \mathrm{d} \theta .
$$




\subsection{Magnetization mechanisms}

For magnetic materials three kinds of magnetization mechanisms can be observed: domain wall bowing, domain wall motion, and domain rotation. Domain wall bowing is treated in this model as reversible kind of domain wall motion. Magnetization for each magnetic field value and angle can be calculated as integral (3):

$$
\begin{aligned}
\boldsymbol{m} & =\boldsymbol{\Gamma}(\boldsymbol{u})(\theta)=\iint_{\alpha \geq \beta} \mu(\alpha, \beta \\
& \times \gamma_{\alpha \beta}\left[\boldsymbol{H}_{\|}\right](\theta) \boldsymbol{\chi}_{\alpha \beta}\left[\boldsymbol{H}_{\chi}\right](\theta) \mathrm{d} \alpha \mathrm{d} \beta .
\end{aligned}
$$

where $\gamma_{\alpha \beta}\left[\boldsymbol{H}_{\|}\right](\theta)$ is switching operator and $\boldsymbol{\chi}_{\alpha \beta}\left[\boldsymbol{H}_{\chi}\right](\theta)$ is rotational operator.

\subsection{Domain wall movement}

When magnetic field increases, domains oriented in magnetic field direction grow and domains in opposite direction shrink proportionally. Spins are switching to opposite directions. Domain movement occurs when field is parallel to domain direction. Thus projection of magnetic field on the Preisach plane is responsible for switching process. Switching magnetic field can be calculated as $(4)$ :

$$
H_{\|}(\theta)=\|\boldsymbol{H}\| \cos \theta \text {. }
$$

Only spins which have lower $\beta$ value or greater $\alpha$ value than projection of magnetic field switch to opposite value. Other spins remain in their previous state $\gamma_{\text {prev }}$ (5) (Fig. 1):

$$
\gamma_{\alpha \beta}\left[\boldsymbol{H}_{\|}(\theta)=\left\{\begin{array}{l}
+1, \quad H_{\|}(\theta)>\alpha \\
\gamma_{\text {prev }}, \quad H_{\|}(\theta)<\alpha \\
-1, \quad H_{\|}(\theta)<\beta
\end{array}\right.\right.
$$

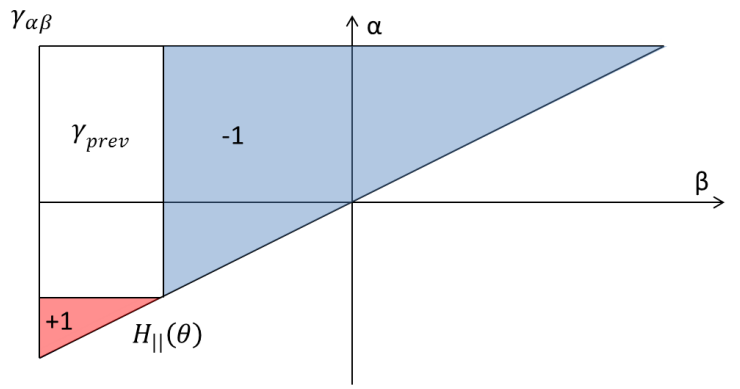

Fig. 1. Switching operator for different field values.

\subsection{Domain rotation}

Domain rotation is reversible process. Thus magnetisation of the Preisach plane should be similar like for anhysteretic curve achieved by demagnetization process (Fig. 2). This process does not depend on domain sign, but on only absolute value of sinus of angle between domain direction and external field direction. Domain rotates to another cross-section plane, so rotational operator can have 1 or 0 value. Rotational magnetic field for each Preisach plane can be calculated as (6):

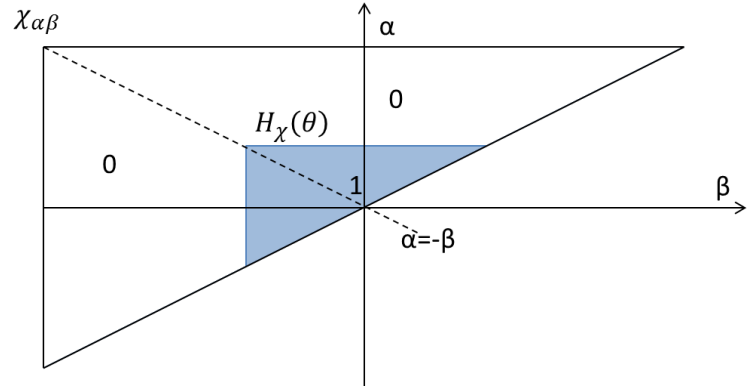

Fig. 2. Rotational operator for different field values.

$$
H_{\mid \chi}(\theta)=H_{\text {sat }}-\|\boldsymbol{H}\||\sin \theta|
$$

Domain rotational operator is defined by the formula (7):

$$
\chi_{\alpha \beta}\left[\boldsymbol{H}_{\chi}\right](t)= \begin{cases}0 & : H_{\chi}(\theta)>\alpha \vee H_{\chi}(\theta)<\beta, \\ 1 & : H_{\chi}(\theta)<\alpha \wedge H_{\chi}(\theta)>\beta .\end{cases}
$$

As a consequence of that rotational operator description is that for $\sin \theta=0$ rotation mechanism has no influence on the Preisach plane. For $H=0$ every domain direction has the same probability. Both motion and rotation mechanisms operate on the same Preisach planes. They have no influence on distribution shape.

\subsection{Anisotropy}

Anisotropy of magnetic properties has influence on hysteresis shape for different directions of magnetization. Differences can be observed in different slope of hysteresis but the same magnetization saturation and coercivity. Thus in presented model only distribution width $\sigma_{c}$ and $\sigma_{i}$ changes for different angles. Uniaxial anisotropy of distribution parameters was assumed, which is common for magnetic materials [10]. Therefore anisotropy coefficient can be expressed as (8):

$$
\alpha_{\phi}=1-|a|+a \sin ^{2} \phi,
$$

where $a$ is anisotropy strength, $\phi$ is distribution angle. $\alpha_{\phi}$ reaches values between -1 and 1 . Easy axis is for angle $\phi=0$, at which $\alpha_{\phi}$ has the lowest absolute value. For $a=1$ or -1 anisotropy is highest, for $a=1$ material is isotropic. Actual distribution width for given angle is therefore $(9),(10)$ :

$$
\begin{aligned}
& \sigma_{c}(\phi)=\alpha_{\phi} \sigma_{c} \\
& \sigma_{i}(\phi)=\alpha_{\phi} \sigma_{i}
\end{aligned}
$$

This approach enables modelling real anisotropy confirmed by experiments, which cannot be achieved by $w$ parameter described in [11]. Anisotropy (8) can be also described by other equation according to given material.

\subsection{Magnetization characteristics}

Presented model has five parameters. For isotropic material $a=1$, thus only four parameters are needed. To verify the model, its ability to model isotropic material was investigated. $\mathrm{Mn}-\mathrm{Zn}$ power ferrite ring shaped was tested. Ring shaped core was approximated by infinite 
rod. Simulated magnetization direction was in fact direction parallel to closed circular field generated by windings. Tested core was sufficiently simulated. The Pearson coefficient $r^{2}=0.998$ was achieved (Fig. 3).

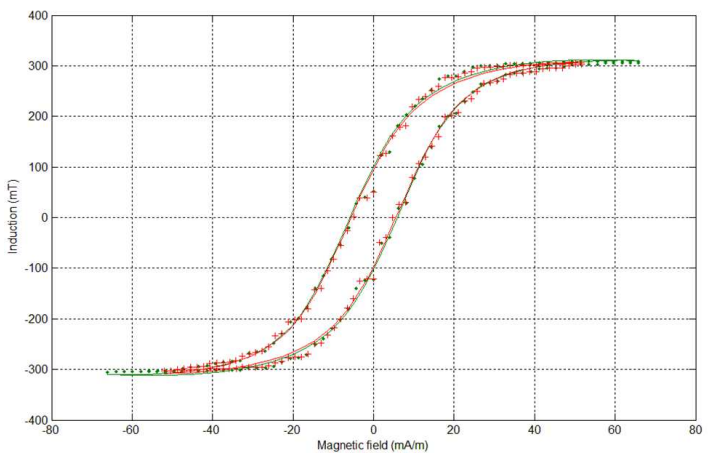

Fig. 3. Modeling results for $\mathrm{Mn}-\mathrm{Zn}$ power ferrite. Symbols - model, line - measured data.
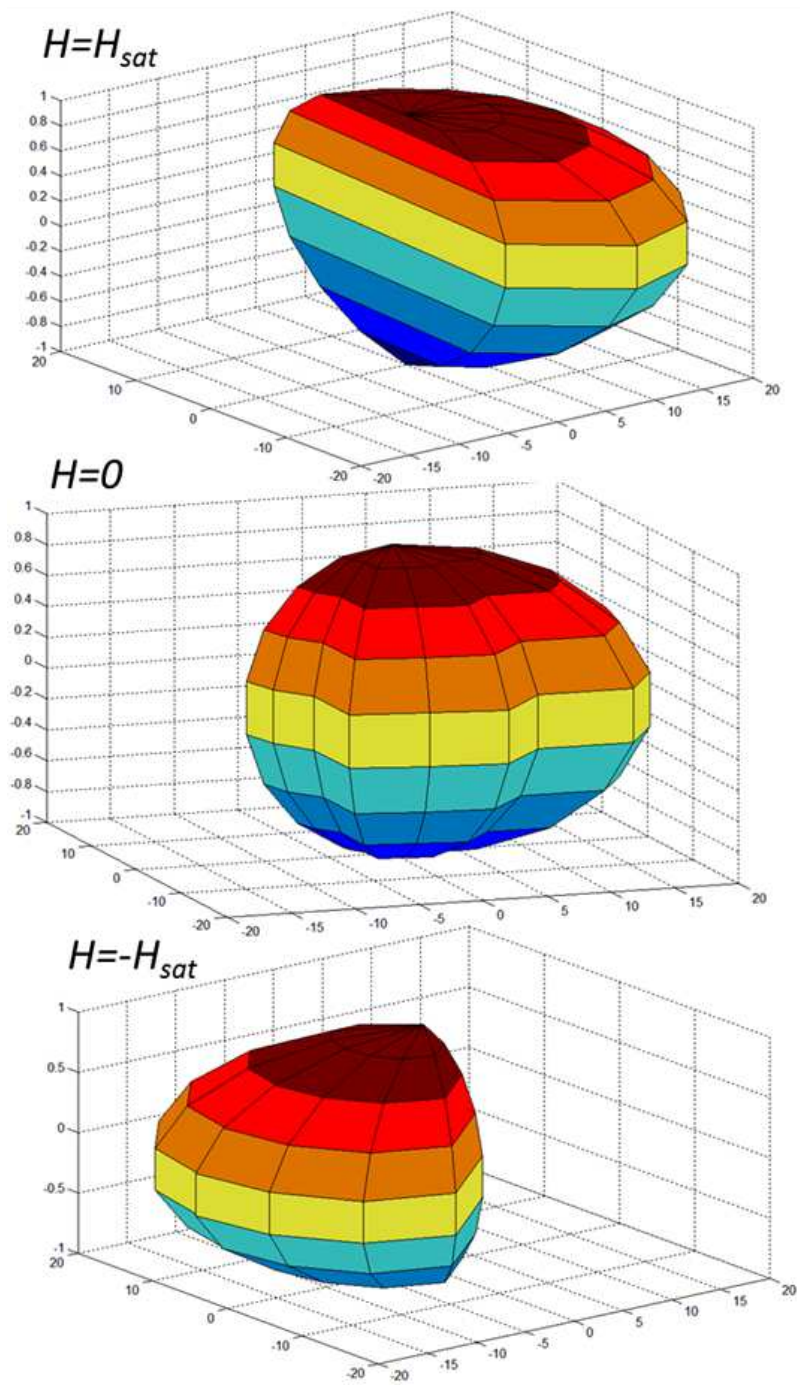

Fig. 4. Magnetization distribution in anisotropic material.
For major hysteresis loop and three field values angular magnetization distribution in anisotropic material was simulated (Fig. 4).

\section{Conclusions}

Presented model needs to be tested for actual anisotropic materials and different angles between easy axis and external magnetic field. Nonetheless, developed model shows new approach to the Preisach model. It consists of three components: magnetization mechanisms, anisotropy and switching operators distribution. It enables further development and easy adaptation for different materials. Presented model simulates complete distribution of magnetic vectors in every direction and has only five parameters for anisotropic materials, and four parameters for isotropic materials.

\section{References}

[1] Chang Seop Koh, Soo Yoon, Ki Woong Nam, Hong Soon Choi, IEEE Trans. Magn. 33, 1822 (1997).

[2] H. Kikuchi, I. Shimizu, K. Sato, K. Iwata, Case Stud. Nondestruct. Test. Evaluat. 1, 25 (2014).

[3] M. Kachniarz, D. Jackiewicz, M. Nowicki, A. Bieńkowski, R. Szewczyk, W. Winiarski, Mechatronics Ideas for Industrial Application, Eds. J. Awrejcewicz, R. Szewczyk, M. Trojnacki, M. Kaliczyńska, Springer Int. Publ., 2015, p. 307.

[4] G. Bertotti, Hysteresis in Magnetism, Academic Press, San Diego 1998, p. 205.

[5] P. Frydrych, R. Szewczyk, M. Nowicki, in: Challenges in Automation, Robotics and Measurement Techniques, Eds. R. Szewczyk, C. Zieliński, M. Kaliczyńska, p. 827.

[6] A. Sutor, S. Bi, R. Lerch, Appl. Phys. 118, 939 (2015).

[7] I.D. Mayergoyz, G. Fiedman, IEEE Trans. Magn. 24, 2928 (1988).

[8] P. Frydrych, R. Szewczyk, Recent Adv. Automat. Robot. Measur. Techn. 267, 591 (2014).

[9] V. Basso, G. Bertotti, A. Infortuna, M. Pasquale, IEEE Trans. Magn. 31, 4000 (1995).

[10] G. Bertotti, Hysteresis in Magnetism, Academic Press, San Diego 1998, p. 146.

[11] M. Kuczmann, J. Adv. Res. Phys. 1, 011003 (2010). 